International Journal of Pure and Applied Mathematics

Volume 109 No. $3 \quad 2016,557-571$

ISSN: 1311-8080 (printed version); ISSN: 1314-3395 (on-line version)

url: http://www.ijpam.eu

doi: 10.12732/ijpam.v109i3.6

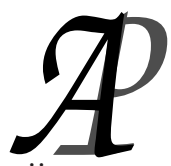

\title{
NEW EXPLICIT TRIGONOMETRICALLY-FITTED FOURTH-ORDER AND FIFTH-ORDER RUNGE-KUTTA-NYSTRÖM METHODS FOR PERIODIC INITIAL VALUE PROBLEMS
}

\author{
M.A. Demba ${ }^{1}$, N. Senu ${ }^{2}$, F. Ismail ${ }^{3}$ \\ ${ }^{1}$ Department of Mathematics \\ Universiti Putra Malaysia \\ 43400 UPM Serdang, Selangor, MALAYSIA \\ ${ }^{2,3}$ Department of Mathematics \\ Institute for Mathematical Research \\ Universiti Putra Malaysia \\ 43400 UPM Serdang, Selangor, MALAYSIA
}

\begin{abstract}
In this work, we derive two new Runge-Kutta-Nyström (RKN) methods for solving periodic second order ordinary differential equations. We constructed these methods based on the RKN method of three-stage fourth-order derived by Garcia and RKN method of four-stage fifth-order derived by Hairer. The numerical results show that the efficiency of the new methods is more than the other existing methods.
\end{abstract}

AMS Subject Classification: 65L05, 65L06

Key Words: trigonometric fitting, RKN methods, periodic initial value problems

\section{Introduction}

In this work, our main focus is to find the numerical solution of the initial value

Received:

May 22, 2016

Revised:

July 5, 2016

Published: September 30, 2016

$\S_{\text {Correspondence author }}$ (c) 2016 Academic Publications, Ltd. url: www.acadpubl.eu 
problems whose first derivative does not appear explicitly of the form:

$$
\begin{gathered}
y^{\prime \prime}=f(x, y), \quad x \in\left[x_{0}, X\right], \\
y\left(x_{0}\right)=y_{0}, \quad y^{\prime}\left(x_{0}\right)=y_{0}^{\prime},
\end{gathered}
$$

whose solution has a pronounced periodic character. Such problems occur in several fields of applied sciences such as: dynamics, celestial mechanics, theoretical physics, nuclear physics, electronics, chemistry and so on; Which attracts the interest of many researchers. Bettis in [3] suggested the first Runge-Kutta (RK) methods with 3 and 4 stages for the solution of Ordinary Differential Equations (ODEs) with oscillatory solutions. Researchers have proposed to develop integrators with frequency-dependent coefficients by some techniques like trigonometrical/exponential fitting as in Simos and Aguiar in [20] and Simos in [19], Franco in [7], Van de Vyver in [22]. Early presentations of these technique are due to Gautschi in [9] and Lyche in [11]. More recently, Monovasilis et al in [12], Franco and Gomez in [6], D'Ambrosio et al in [5], Ramos and Aguiar in [14] proposed Runge-Kutta-Nyström (RKN) methods for the solution of second order ordinary differential equations. Similarly, Simos in [17], Coleman and Duxbury in [4] constructed an explicit RK method which integrate certain first order initial value problems with periodic or oscillating solutions. In the same manner, Berghe et al in [2] proposed exponentially-fitted RK (EFRK) methods that integrates exactly first order systems whose solutions can be represented as the linear combination of functions of the form $e^{w x}$ and $e^{-w x}$. Motivated by the work of Simos in [18] we construct an explicit trigonometrically-fitted fourth-order three stage and fifth-order four stage RKN methods based on the RKN method derived by Garcia et al in [8] and an RKN method derived by Hairer and Wanner in [10]. The remaining part of this paper is arranged as follows: Section 2 deals with the basic theory of an explicit RKN method, definition of trigonomeric-fitting. In section 3 we construct the new methods. In section 4 we analyze the algebraic order of the new methods from their local truncation errors. In section 5 we present the numerical results and the last section deals with the conclusion.

\section{Basic Theory}

A Runge-Kutta-Nyström method is said to be explicit if $a_{i j}=0$, for $i \leq j$. The general form of an explicit $s$-stage RKN method is given by: 


$$
\begin{gathered}
y_{n+1}=y_{n}+h y_{n}^{\prime}+h^{2} \sum_{i=1}^{s} b_{i} f\left(x_{n}+c_{i} h, Y_{i}\right), \\
y_{n+1}^{\prime}=y_{n}^{\prime}+h \sum_{i=1}^{s} d_{i} f\left(x_{n}+c i h, Y_{i}\right), \\
Y_{i}=y_{n}+c_{i} h y_{n}^{\prime}+h^{2} \sum_{j=1}^{i-1} a_{i j} f\left(x_{n}+c i h, Y_{j}\right) .
\end{gathered}
$$

or in Butcher Tableau as:

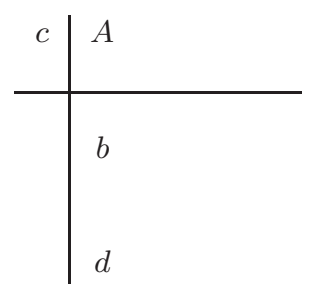

where $\mathrm{A}$ is a matrix $\left(a_{i j}\right)_{s \times s}, c=\left(c_{1}, c_{2}, \ldots, c_{s}\right)^{T}, b=\left(b_{1}, b_{2}, \ldots, b_{s}\right)^{T}$ and $d=\left(d_{1}, d_{2}, \ldots, d_{s}\right)^{T}$.

Definition 1. A Runge-Kutta-Nyström method (2)-(4) is said to be trigonometrically-fitted if it integrates exactly the function $e^{i w x}$ and $e^{-i w x}$ or equivalently $\sin (w x)$ and $\cos (w x)$ with $w>0$ the principal frequency of the problem when applied to the test equation $y^{\prime \prime}=-w^{2} y$; Leading to a system of equations as derived in the next section.

\section{Derivation of the New Methods}

In this section, we will derive a three-stage fourth order and a four-stage fifth order explicit trigonometrically-fitted RKN methods. The three-stage fourthorder RKN method will be used as given in [8] and a four stage fifth-order RKN method will be used as given in [10]. The coefficients of the methods are given in Table 1 and Table 2 below:

When an explicit Runge-Kutta-Nyström method (2) - (4) is applied to the test equation $y^{\prime \prime}=-w^{2} y$, the method become: 
Table 1: The RKN4G Method [8]

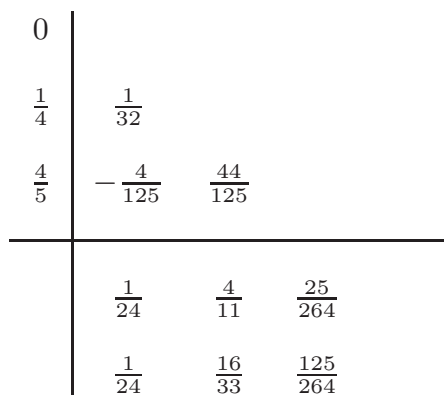

Table 2: The RKN5H Method [10]

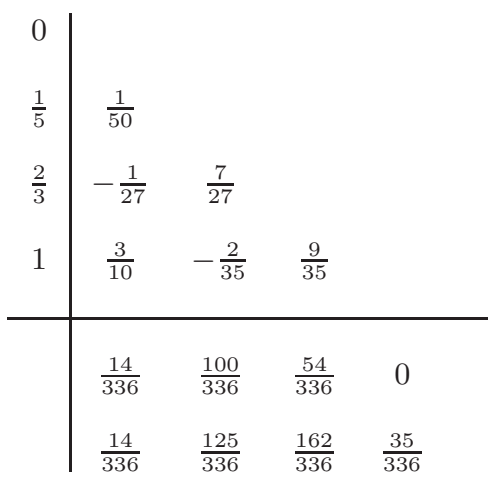

$$
\begin{aligned}
& y_{n+1}=y_{n}+h y_{n}^{\prime}+h^{2} \sum_{i=1}^{s} b_{i} f\left(x_{n}+c_{i} h, Y_{i}\right), \\
& y_{n+1}^{\prime}=y_{n}^{\prime}+h \sum_{i=1}^{s} d_{i} f\left(x_{n}+c i h, Y_{i}\right),
\end{aligned}
$$

with

$$
\begin{aligned}
& Y_{1}=y_{n}+c_{1} h y_{n}^{\prime}, \\
& Y_{2}=y_{n}+c_{2} h y_{n}^{\prime}-h^{2} a_{21} w^{2} Y_{1}, \\
& Y_{3}=y_{n}+c_{3} h y_{n}^{\prime}+h^{2}\left(-a_{31} w^{2} Y_{1}-a_{32} w^{2} Y_{2}\right),
\end{aligned}
$$




$$
\begin{aligned}
& Y_{4}=y_{n}+c_{4} h y_{n}^{\prime}+h^{2}\left(-a_{41} w^{2} Y_{1}-a_{42} w^{2} Y_{2}-a_{43} w^{2} Y_{3}\right), \\
& \cdot \\
& \cdot \\
& Y_{i}=y_{n}+c_{i} h y_{n}^{\prime}+h^{2} \sum_{j=1}^{s} a_{i j}\left(-w^{2} Y_{j}\right),
\end{aligned}
$$

which results in

$$
y_{n+1}=y_{n}+h y_{n}^{\prime}+h^{2} \sum_{i=1}^{s} b_{i}\left(-w^{2} Y_{i}\right) \text {, }
$$

and

$$
y_{n+1}^{\prime}=y_{n}^{\prime}+h \sum_{i=1}^{s} d_{i}\left(-w^{2} Y_{i}\right) .
$$

Now, let $y_{n}=e^{I w x}$. Computing the value of $y_{n+1}, y_{n}^{\prime}$ and $y_{n+1}^{\prime}$ and substituting in the equations $(5)-(13)$ and by using $e^{I v}=\cos (v)+I \sin (v)$ and comparing the real and imaginary part, we obtain the following system of equations:

$$
\begin{aligned}
& \cos (v)=1-v^{2} \sum_{i=1}^{s} b_{i}\left(1-v^{2} \sum_{j=1}^{3} a_{i j} Y_{j} e^{-I w x}\right) \\
& \sin (v)=v-v^{2} \sum_{i=1}^{s} b_{i} c_{i} v \\
& \sin (v)=v \sum_{i=1}^{s} d_{i}\left(1-v^{2} \sum_{j=1}^{3} a_{i j} Y_{j} e^{-I w x}\right) \\
& \cos (v)=1-v^{2} \sum_{i=1}^{s} d_{i} c_{i} .
\end{aligned}
$$

where $v=w h$.

Solving (14)-(17) using the coefficients of the method in Table 1 for $a_{31}, b_{2}, c_{2}, c_{3}$, we obtain the solution as given in (18):

$$
\begin{aligned}
a_{31} & =-\frac{2112 \sin (v)-2112 v+384 v^{3}-11 v^{5}}{1000 v^{3}}, \\
b_{2} & =\frac{16\left(330 \cos (v)-330-21 v^{2}+66 v \sin (v)+v^{4}\right)}{165\left(v^{2}-32+v^{2}\right)},
\end{aligned}
$$




$$
\begin{aligned}
c_{2}= & \frac{3(\cos (v)+4)\left(-160 \cos (v) \sin (v)+5 \cos (v) \sin (v) v^{2}-480 v-32 v \sin (v)^{2}+15 v^{3}\right.}{16\left(\left(\sin (v)^{2}+15\right) v\left(v^{2}+30 \cos (v)-30+6 v \sin (v)\right)\right)} \\
& +\frac{\left.v^{3} \sin (v)^{2}+640 \sin (v)-20 \sin (v) v^{2}\right)}{16\left(\left(\sin (v)^{2}+15\right) v\left(v^{2}+30 \cos (v)-30+6 v \sin (v)\right)\right)}, \\
c_{3}= & \frac{3\left(2112 v \sin (v)^{3}-63360 \sin (v)^{2}-672 v^{2} \sin (v)^{2}+384 v^{4} \sin (v)^{2}-11 v^{6} \sin (v)^{2}\right)}{500\left(\sin (v)^{2}+15\right)\left(v^{2}+30 \cos (v)-30+6 v \sin (v)\right) v^{2}} \\
& +\frac{3\left(10560 \cos (v) \sin (v)^{2}+5440 \cos (v) \sin (v) v+9920 v \sin (v)-7680 \sin (v) v^{3}\right.}{500\left(\sin (v)^{2}+15\right)\left(v^{2}+30 \cos (v)-30+6 v \sin (v)\right) v^{2}} \\
& +\frac{1920 \cos (v) \sin (v) v^{3}-55 \cos (v) v^{5} \sin (v)+220 v^{5} \sin (v)+5760 v^{4}+1760 v^{2} \cos (v)}{500\left(\sin (v)^{2}+15\right)\left(v^{2}+30 \cos (v)-30+6 v \sin (v)\right) v^{2}} \\
& -\frac{\left.17120 v^{2}-105600 \cos (v)+105600-165 v^{6}\right)(\cos (v)+4)}{500\left(\sin (v)^{2}+15\right)\left(v^{2}+30 \cos (v)-30+6 v \sin (v)\right) v^{2}} .
\end{aligned}
$$

The corresponding Taylor series expansion of the solution is given in (19).

$$
\begin{aligned}
a_{31}= & -\frac{4}{125}-\frac{33}{5000} v^{2}+\frac{11}{26250} v^{4}-\frac{11}{1890000} v^{6}+\frac{1}{18900000} v^{8}-\frac{1}{2948400000} v^{10} \\
+ & \frac{1}{619164000000} v^{12}-\frac{1}{168412608000000} v^{14}+\ldots, \\
b_{2}= & \frac{4}{11}-\frac{1}{3600} v^{4}+\frac{1}{161280} v^{6}-\frac{19}{232243200} v^{8}+\frac{449}{1226244096000} v^{10}-\frac{32869}{3570822807552000} v^{12} \\
& -\frac{312811}{1713994947624960000} v^{14}+\ldots, \\
c_{2}= & \frac{1}{4}-\frac{1}{26880} v^{4}+\frac{19}{7741440} v^{6}-\frac{1019}{13624934400} v^{8}+\frac{481093}{119027426918400} v^{10} \\
- & \frac{8198117}{57133164920832000} v^{12}+\frac{647998097}{93241325150797824000} v^{14}+\ldots, \\
c_{3}= & \frac{4}{5}+\frac{13}{4375} v^{4}-\frac{257}{3780000} v^{6}+\frac{1447}{950400000} v^{8}-\frac{2026831}{58118860800000} v^{10} \\
+ & \frac{6352153}{3985293312000000} v^{12}+\ldots
\end{aligned}
$$

This result in the new method called ETFRKN4G.

As $v \rightarrow 0$, the coefficients $a_{31}, b_{2}, c_{2}$ and $c_{3}$ of the new method (ETFRKN4G) reduces to the coefficients of the original method (RKN4G). That is to say $a_{31}(0), b_{2}(0), c_{2}(0)$ and $c_{3}(0)$ are identical to $a_{31}, b_{2}, c_{2}$ and $c_{3}$ of RKN4G method. In a similar way, solving (14)-(17) using the coefficients of the method in Table 2 for $a_{31}, b_{2}, c_{2}, c_{3}$, obtaining the solution as given in (20).

$$
a_{31}=\frac{50400 \sin (v)-470 v^{5}+7 v^{7}+9300 v^{3}-50400 v}{v^{3}\left(v^{2}-18\right)},
$$




$$
\begin{aligned}
b_{2}= & -\frac{-4200 \cos (v) v^{2}+75600 \cos (v)-5700 v^{2}-75600+650 v^{4}+25200 v \sin (v)+3 v^{6}}{84\left(1350 v^{2}\left(-68 v^{2}+900+v^{4}\right)\right)} \\
c_{2}= & \frac{3\left(-3 v^{5}+8 \sin (v) v^{4}+48 v^{3} \cos (v)+246 v^{3}-544 \sin (v) v^{2}-4800 v-2400 v \cos (v)+7200 \sin (v)\right)}{25 v\left(7 v^{4}+42 v^{2}-48 \cos (v) v^{2}+288 v \sin (v)-864+864 \cos (v)\right)} \\
c_{3}= & \frac{14515200-816000 \sin (v) v^{3}+56 v^{9} \sin (v)+336 v^{8} \cos (v)+612000 \cos (v) v^{2}}{225 v^{2}\left(v^{2}-18\right)\left(7 v^{4}+42 v^{2}-48 \cos (v) v^{2}+288 v \sin (v)-864+864 \cos (v)\right)} \\
& -\frac{7257600 \sin (v)^{2}+2419200 \cos (v) \sin (v) v+280800 v \sin (v)+2082 v^{8}}{225 v^{2}\left(v^{2}-18\right)\left(7 v^{4}+42 v^{2}-48 \cos (v) v^{2}+288 v \sin (v)-864+864 \cos (v)\right)} \\
& +\frac{403200 v^{2} \sin (v)^{2}-14515200 \cos (v)-21 v^{10}-3312000 v^{2}+646200 v^{4}-58120 v^{6}}{225 v^{2}\left(v^{2}-18\right)\left(7 v^{4}+42 v^{2}-48 \cos (v) v^{2}+288 v \sin (v)-864+864 \cos (v)\right)} \\
& +\frac{118680 \sin (v) v^{5}+322800 v^{4} \cos (v)-4768 v^{7} \sin (v)-22560 v^{6} \cos (v)}{225 v^{2}\left(v^{2}-18\right)\left(7 v^{4}+42 v^{2}-48 \cos (v) v^{2}+288 v \sin (v)-864+864 \cos (v)\right)}
\end{aligned}
$$

The corresponding Taylor series expansion of the solution is given in (21):

$$
\begin{aligned}
a_{31}= & -\frac{1}{27}+\frac{1}{8100} v^{4}+\frac{1}{874800} v^{6}+\frac{1}{8660520} v^{8}+\frac{493}{81062467200} v^{10}+\frac{197}{580333572000} v^{12} \\
& +\frac{2356967}{125017779414528000} v^{14}+\ldots, \\
b_{2}= & \frac{25}{84}+\frac{1}{1800} v^{4}+\frac{31}{5040000} v^{6}+\frac{283}{486000000} v^{8}+\frac{1893851}{67359600000000} v^{10} \\
+ & \frac{534849793}{344796952500000000} v^{12}+\frac{77213533763}{902741112000000000000} v^{14}+\ldots, \\
c_{2}= & \frac{1}{5}+\frac{11}{21875} v^{4}+\frac{661}{13125000} v^{6}+\frac{388123}{97453125000} v^{8}+\frac{1783503283}{5320940625000000} v^{10} \\
+ & \frac{11783318681}{399070546875000000} v^{12}+\frac{3240032263921}{1252467562500000000000} v^{14}+\ldots, \\
c_{3}= & \frac{2}{3}-\frac{11}{28350} v^{4}+\frac{157}{12757500} v^{6}+\frac{94709}{28066500000} v^{8}+\frac{7150813}{39181471875000} v^{10} \\
+ & \frac{39653233271}{3103172572500000000} v^{12}+\ldots
\end{aligned}
$$

This result in the new method called ETFRKN5H.

As $v \rightarrow 0$, the coefficients $a_{31}, b_{2}, c_{2}$ and $c_{3}$ of the new method (ETFRKN5H) reduces to the coefficients of the original method (RKN5H). That is to say $a_{31}(0), b_{2}(0), c_{2}(0)$ and $c_{3}(0)$ are identical to $a_{31}, b_{2}, c_{2}$ and $c_{3}$ of RKN5H method.

\section{Algebraic Order and Error Analysis}

In this section, we will find the local truncation error of the new methods and verify their algebraic order. We first find the Taylor series expansion of the 
actual solution $y\left(x_{n}+h\right)$, the first derivative of the actual solution $y^{\prime}\left(x_{n}+h\right)$, the approximate solution $y_{n+1}$, and the first derivative of the approximate solution $y_{n+1}^{\prime}$. The local truncation error (LTE) of y and its first derivative $y^{\prime}$ is given as:

$$
\begin{aligned}
L T E & =y_{n+1}-y\left(x_{n}+h\right), \\
L T E_{\text {der }} & =y_{n+1}^{\prime}-y^{\prime}\left(x_{n}+h\right) .
\end{aligned}
$$

The $L T E$ and $L T E_{d e r}$ of the ETFRKN4G method are

$$
\begin{aligned}
L T E & =-\frac{h^{5}}{480}\left(y^{\prime} y^{\prime \prime} f_{y y}+y^{\prime \prime} f_{x y}+y^{\prime} f_{y x x}+\left(y^{\prime}\right)^{2} f_{x y y}\right) \\
& -\frac{h^{5}}{1440}\left(\left(y^{\prime}\right)^{3} f_{y y y}+f_{x x x}\right)+O\left(h^{6}\right), \\
L T E_{d e r} & =\frac{h^{5}}{120}\left(6\left(y^{\prime}\right)^{2} y^{\prime \prime} f_{y y y}+5\left(y^{\prime}\right)^{2} f_{y y} f_{y}+12 y^{\prime} y^{\prime \prime} f_{x y y}+6 y^{\prime} f_{x y} f_{y}+4 y^{\prime} f_{y y} f_{x}\right. \\
& +\left(y^{\prime}\right)^{4} f_{y y y y}+4 y^{\prime} f_{x x x y}+y^{\prime \prime}\left(f_{y}\right)^{2}+\left(y^{\prime}\right)^{2} f_{x x y y}+6\left(y^{\prime}\right)^{2} f_{x x y y}+f_{y} f_{x} \\
& \left.+4 f_{x y} f_{x}+4\left(y^{\prime}\right)^{3} f_{x y y y}+6 y^{\prime \prime} f_{y x x}+f_{x x x x}+3\left(y^{\prime \prime}\right)^{2} f_{y y}\right)+O\left(h^{6}\right) .
\end{aligned}
$$

From equation (23), we can see that the order of ETFRKN4G is 4 because all of the coefficients up to $h^{4}$ vanished. Similarly, the LTE and LTE der of the method ETFRKN5H are

$$
\begin{aligned}
L T E & =\frac{h^{6}}{21600}\left(-12 w^{4} y^{\prime \prime}+6\left(y^{\prime}\right)^{2} y^{\prime \prime} f_{y y y}+12\left(y^{\prime}\right)^{2} f_{y y} f_{y}+12 y^{\prime} y^{\prime \prime} f_{x y y}\right. \\
& +24 y^{\prime} f_{y} f_{x y}+3\left(y^{\prime \prime}\right)^{2} f_{y y}+12\left(f_{y}\right)^{2} y^{\prime \prime} \\
& +4\left(y^{\prime}\right)^{3} f_{x y y y}+\left(y^{\prime}\right)^{4} f_{y y y y}+12 f_{y} f_{x x}+6 y^{\prime \prime} f_{y x x} \\
& \left.+6\left(y^{\prime}\right)^{2} f_{x x y y}+4 y^{\prime} f_{x x x y}+f_{x x x x}\right)+O\left(h^{7}\right), \\
L T E_{d e r} & =\frac{h^{6}}{720}\left(5 y^{\prime} f_{x x x x y}+10 y^{\prime \prime} f_{x x x y}+\left(f_{y}\right)^{2} f_{x}+5 f_{x y} f_{x x}+10 y^{\prime}\left(f_{x y}\right)^{2}\right. \\
& +f_{y} f_{x x x}+15\left(y^{\prime \prime}\right)^{2} f_{x y y}+y^{\prime}\left(f_{y}\right)^{3} \\
& +5\left(y^{\prime}\right)^{4} f_{x y y y y}+10\left(y^{\prime}\right)^{2} f_{x x x y y}+10\left(y^{\prime}\right)^{3} f_{x x y y y} \\
& +10 f_{x x x x x}+11\left(y^{\prime}\right)^{3} f_{y y y} f_{y}+10\left(y^{\prime}\right)^{2} f_{y y y} f_{x} \\
& +30\left(y^{\prime}\right)^{2} y^{\prime \prime} f_{x y y y}+30 y^{\prime} f_{x x y y} y^{\prime \prime}+15 y^{\prime} f_{y y}\left(y^{\prime \prime}\right)^{2} \\
& +f_{x x x x}+23\left(y^{\prime}\right)^{2} f_{y} f_{x y y}+20 y^{\prime} f_{x} f_{x y y}+8 f_{y} f_{x y} y^{\prime \prime} \\
& +10 f_{y y y y} y^{\prime \prime}+10 y^{\prime \prime} f_{y y} f_{x}+13 f_{y} f_{y x x} y^{\prime} \\
& \left.+15\left(y^{\prime}\right)^{2} f_{y y} f_{x y}+5 y^{\prime} f_{y y} f_{x x}\right)+O\left(h^{7}\right) .
\end{aligned}
$$


From equation (24), the method ETFRKN5H has order 5 because all of the coeffients up to $h^{5}$ vanished.

\section{Problems Tested and Numerical Results}

In this section, we will apply the new methods to some well-known periodic problems. The following explicit RKN methods are used for the numerical comparisons.

Fourth-order:

- ETFRKN4G: The new trigonometrically-fitted RKN method derived in this paper,

- RKN4G: The fourth-order RKN method obtained by Garcia et al in [8] ,

- RKN4-4: The fourth-order RKN method given by Xinyuan Wu et al in [23] and

- PFERKN4P: The fourth-order RKN method with phase-lag order infinity obtained by Papadopoulos in [13].

Fifth-order:

- ETFRKN5H: The new trigonometrically-fitted RKN method derived in this paper,

- RKN5H: The fifth order RKN method obtained by Hairer and Wanner in $[10]$,

- RKN4(5,8,5)S: The fifth order RKN method with dispersion order eight and dissipation order five obtained by Senu in [15] and

- PFERKN4P: The fourth-order RKN method with phase-lag order infinity obtained by Papadopoulos et al in [13].

Problem 1 (Harmonic Oscillator) Anastassi and Kosti in [1]

$$
y^{\prime \prime}=-100 y, y(0)=1, y^{\prime}(0)=2
$$

The exact solution is

$$
y(x)=-\frac{1}{5} \sin (10 x)+\cos (10 x) .
$$

Problem 2 (Inhomogeneous Problem) Anastassi and Kosti in [1] 
$y^{\prime \prime}=-v^{2} y(x)+\left(v^{2}-1\right) \sin (x), y(0)=1, y^{\prime}(0)=v+1, x \geq 0$, where $v>>1$,

The exact solution is

$$
y(x)=\sin (10 x)+\cos (10 x)+\sin (x) .
$$

Problem 3 (Almost Periodic Problem) Van de Vyver in [21]

$$
\begin{aligned}
& y_{1}^{\prime \prime}=-y_{1}+0.001 \cos (x), y_{1}(0)=1, y_{1}^{\prime}(0)=0, \\
& y_{2}^{\prime \prime}=-y_{2}+0.001 \sin (x), y_{2}(0)=0, y_{2}^{\prime}(0)=0.9995
\end{aligned}
$$

The exact solutions are

$$
\begin{aligned}
& y_{1}(x)=\cos (x)+0.0005 x \cos (x), \\
& y_{2}(x)=\sin (x)-0.0005 x \sin (x) .
\end{aligned}
$$

Problem 4 (Inhomogeneous Problem) Senu in [15]

$$
y^{\prime \prime}=-y(x)+x, y(0)=1, y^{\prime}(0)=2
$$

The exact solution is

$$
y(x)=\cos (x)+\sin (x)+x .
$$

Problem 5 (Inhomogeneous System) Senu et al in [16]

$$
\begin{aligned}
& y_{1}^{\prime \prime}=-v^{2} y_{1}(x)+v^{2} f(x)+f^{\prime \prime}(x), y_{1}(0)=a+f(0), y_{1}^{\prime}(0)=f^{\prime}(0), \\
& y_{2}^{\prime \prime}=-v^{2} y_{2}(x)+v^{2} f(x)+f^{\prime \prime}(x), y_{2}(0)=f(0), y_{2}^{\prime}(0)=a v+f^{\prime}(0),
\end{aligned}
$$

The exact solutions are

$$
\begin{aligned}
& y_{1}(x)=a \cos (v x)+f(x), \\
& y_{2}(x)=a \sin (v x)+f(x),
\end{aligned}
$$

where $v=20, a=0.1$ are parameters and $f(x)=e^{-10 x}$.

The accuracy strategy used is finding $\log _{10}$ of the maximum global error (MAXERR),

$$
M A X E R R=\log _{10} \max \left\|y\left(x_{n}\right)-y_{n}\right\|,
$$

where $x_{n}=x_{0}+n h, n=1,2,3, \ldots\left(T-x_{0}\right) / h$.

The performance of the fourth-order method is presented graphically as shown in figures 1 to 5 and the performance of the fifth-order method as in figures 6 to 9 . 


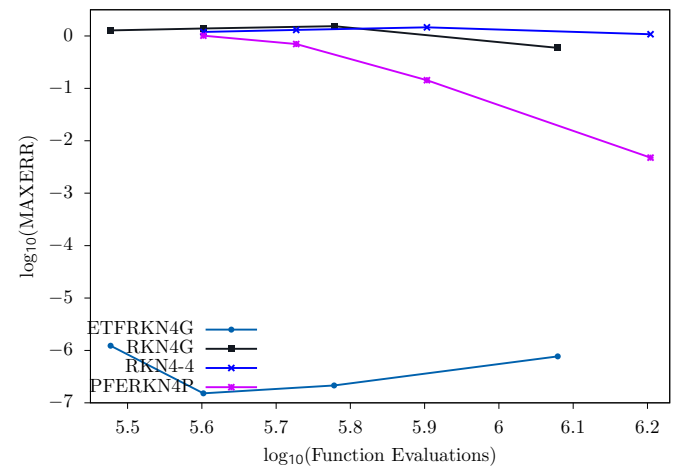

Figure 1: Efficiency curves for problem 1 with $t_{\text {end }}=10000$ and $h=$ $i(0.025), i=1,2,3,4$.

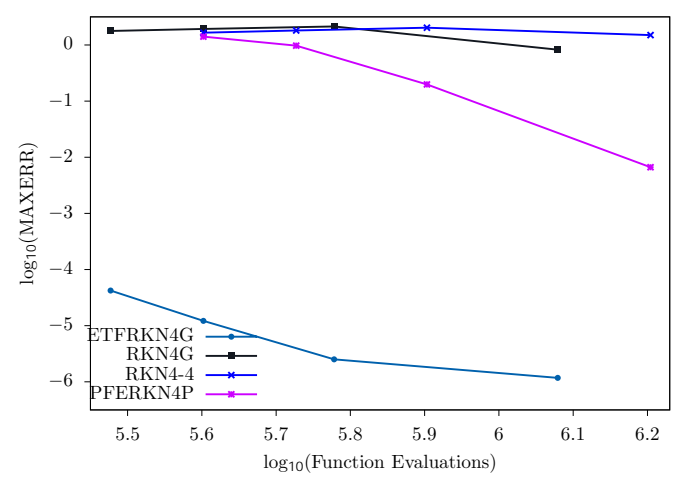

Figure 2: Efficiency curves for problem 2 with $t_{\text {end }}=10000$ and $h=$ $i(0.025), i=1,2,3,4$.

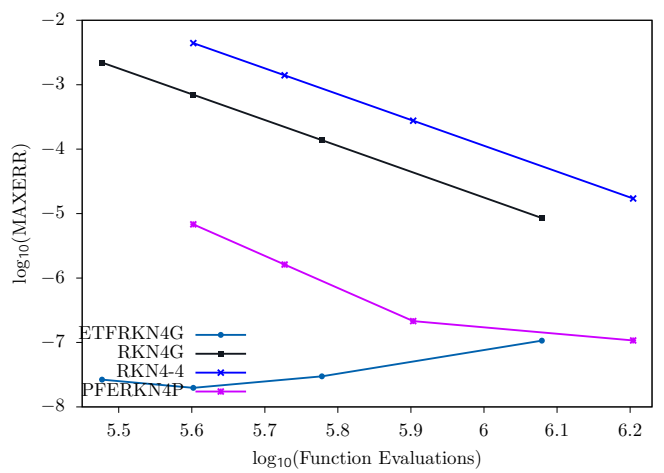

Figure 3: Efficiency curves for problem 3 with $t_{\text {end }}=10000$ and $h=$ $i(0.025), i=1,2,3,4$. 


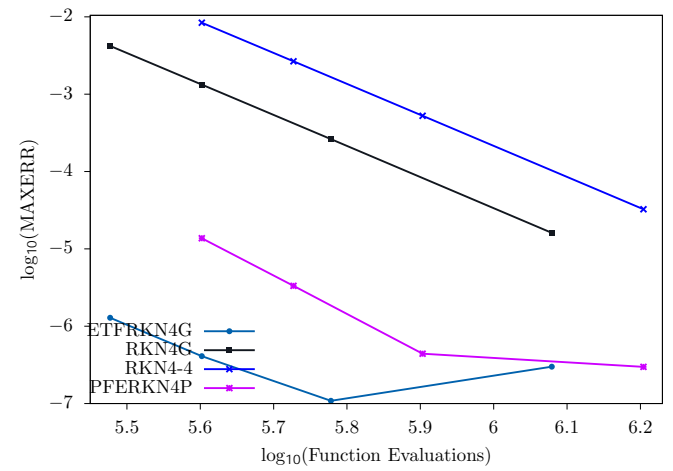

Figure 4: Efficiency curves for problem 4 with $t_{\text {end }}=10000$ and $h=$ $i(0.025), i=1,2,3,4$.

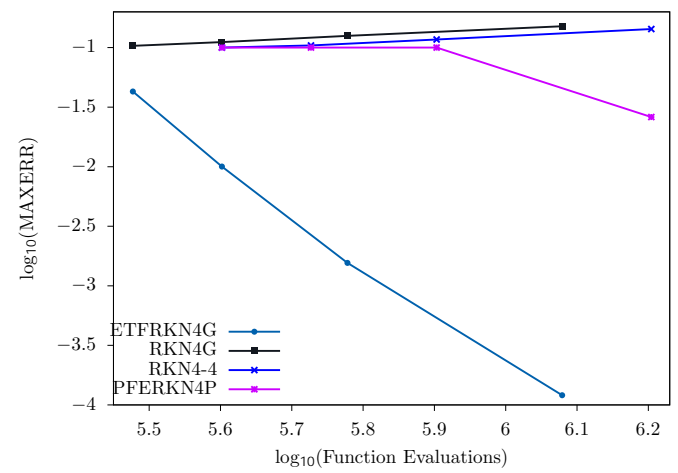

Figure 5: Efficiency curves for problem 5 with $t_{\text {end }}=10000$ and $h=$ $i(0.025), i=1,2,3,4$.

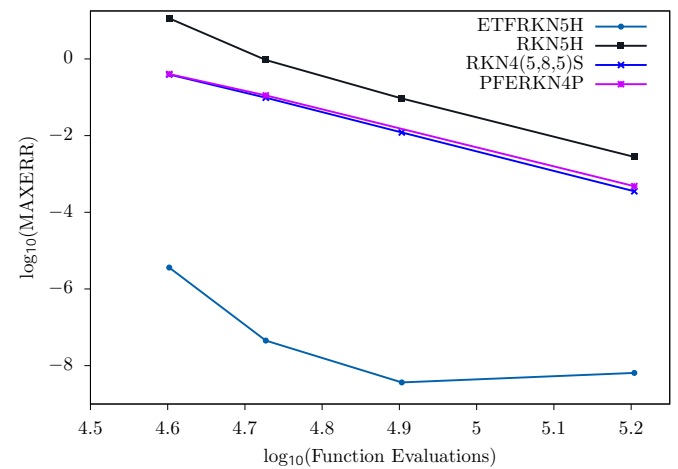

Figure 6: Efficiency curves for problem 1 with $t_{\text {end }}=1000$ and $h=$ $i(0.025), i=1,2,3,4$. 


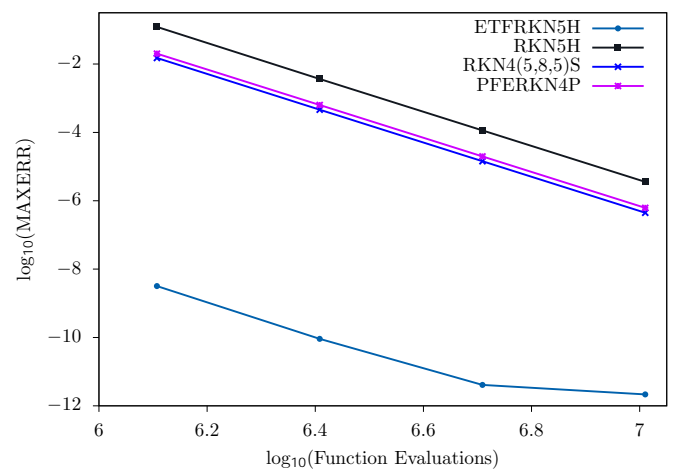

Figure 7: Efficiency curves for problem 2 with $t_{\text {end }}=10000$ and $h=\frac{1}{2^{i}}$, $i=5,6,7,8$.

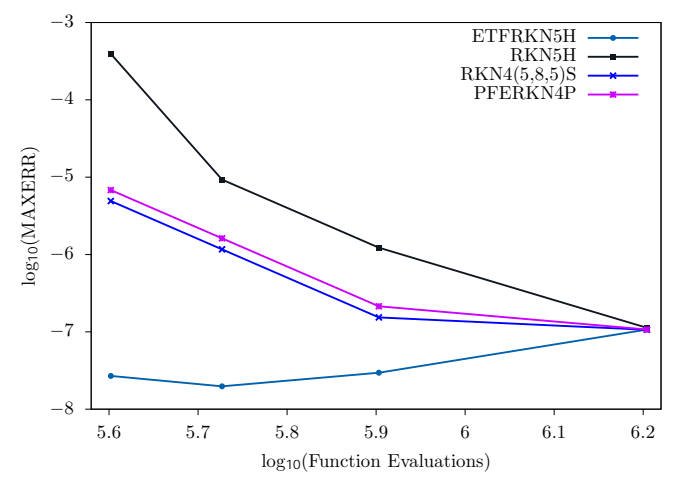

Figure 8: Efficiency curves for problem 3 with $t_{\text {end }}=10000$ and $h=$ $i(0.025), i=1,2,3,4$.

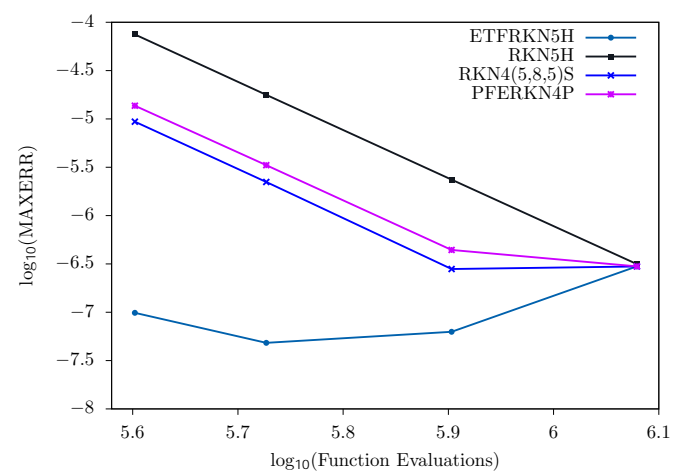

Figure 9: Efficiency curves for problem 4 with $t_{\text {end }}=10000$ and $h=$ $i(0.025), i=1,2,3,4$. 


\section{Conclusion}

In this study, we have presented two new explicit trigonometrically-fitted RKN methods for solving second order IVPs which are periodic in nature. First method is based on Garcia's RKN method of order four and the second method is based on Hairer's RKN method of order five. The numerical results obtained show that the error norm of the new methods are smaller than that of the other existing methods; the new methods are more accurate and efficient for solving second-order differential equations with periodic solutions than the other existing methods.

\section{Acknowledgments}

We are thankful to the referees for their constructive comments and the Institute of Mathematical Research (INSPEM), the Department of Mathematics, Universiti Putra Malaysia for the support and assistance during the research work.

\section{References}

[1] Z. Anastassi and A. Kosti, A 6(4) optimized embedded Runge-Kutta Nyström pair for the numerical solution of periodic problems, Journal of Computational Applied Mathematics, 275 (2013), 311-320.

[2] G. V. Berghe, H. De Meyer, M. Van Daele, and T. Van Hecke Exponentially fitted explicit Runge-Kutta methods,,Computer Physics Communications, 123 (1999), 7-15.

[3] D. Bettis, Runge-Kutta Algorithms for oscillatory problems, Zeitscrift für angewandte Mathematik und Physik ZAMP, 30 (1979), 699-704.

[4] J. P. Coleman and S. C. Duxbury, mixed collocation methods for $y^{\prime \prime}=f(x, y)$, Journal of computational and applied mathematics, 126 (2000), 47-75.

[5] R. D'Ambrosio, B. Paternoster, and G. Santomauro, Revised exponentially fitted RungeKutta-Nyström methods, Applied Mathematics Letters, 30 (2014), 56-60.

[6] J. Franco, and I. Gomez Symplectic explicit methods of Runge-Kutta-Nyström type for solving pertubed oscillators, Journal of Computational and Applied Mathematics, 260 (2014), 482-493.

[7] J. Franco, Runge-Kutta methods adapted to the numerical integration of oscillatory problems, Applied Numerical Mathematics, 50 (2004), 427-443.

[8] A. Garcia, P. Martin, and A. B. Gonzalez et al., New methods for oscillatory problems based on classical codes, Applied Numerical Mathematics, 42 (2002), 141-157.

[9] W. Gautschi, Numerical integration ordinary differential equations based on trigonometric polynomials,,Computer Physics Communications, vol. 115, pp. 1-8, 1998. 
[10] E. Hairer, S. P. Norsett, and G. Wanner, Solving Ordinary Differential Equations I, 2nd edition, Vol. 8, Springer, Berlin Germany, 1993, non stiff Problems.

[11] T. Lyche, Chebyshevian multistep methods for ordinary differential equations, Numerische Mathematik, 19 (1972), 65-75.

[12] T. Monovasilis, Z. Kalogiratou, and T. E. Simos, Exponentially-fitted symplectic RungeKutta-Nyström methods, Appl. Math. Inf. Sci, 7 (2013), 81-85.

[13] D. F. Papadopoulos, Z. Anastassi, A. Zacharias and T. E. Simos, A phase-fitted RungeKutta-Nystrøm method for the numerical solution of initial value problems with oscillating solutions, Computer Physics Communications,vol. 180, pp. 1839-1846, 2009.

[14] H. Ramos and J. Vigo-Aguiar, A trigonometrically-fitted method with two frequencies, one for the solution and another one for the derivative, Computer Physics Communications, 185 (2014), 1-8.

[15] N. Senu, Runge-Kutta-Nystrøm methods for solving oscillatory problems, Ph.D. diss., Department of Mathematics, Faculty of Science, 43400 UPM Serdang, Malaysia, 2009.

[16] N. Senu, M. Suleiman, and F. Ismail, An embedded explicit Runge-Kutta-Nystrøm method for solving oscillatory problems, Physica Scripta, 80 (2009), 015005.

[17] T. E. Simos, An exponentially-fitted Runge-Kutta method for the numerical integration of initial-value problems with periodic or oscillating solutions, Computer Physics Communications, 115 (1998), 1-8.

[18] T. E. Simos, Exponentially-fitted Runge-Kutta-Nystrøm method for the numerical integration of initial-value problems with oscillating solutions, Applied Mathematics Letter, 15 (2002), 217-225.

[19] T. E. Simos, A family of fifth algebraic order trigonometrically-fitted Runge-Kutta methods for the numerical solution of the schrødinger equation, Computational materials science, 34 (2005), 342-354.

[20] T. E. Simos and J. V. Aguiar, A modified phase-fitted Runge-Kutta method for the numerical solution of the schrødinger equation, Journal of mathematical chemistry, 30 (2001), 121-131.

[21] H. Van de Vyver A symplectic exponentially-fitted modified Runge-Kutta-Nystrøm method for the numerical solution of orbital problems, New Astronomy, 10 (2005), 261269.

[22] H. Van de Vyver An embedded exponentially-fitted Runge-Kutta-Nystrøm method for the numerical solution of orbital problems, New Astronomy, 11 (2006), 577-587.

[23] Xinyuan Wu, Xiong You and Bin Wang, Structure preserving algorithms for oscillatory differential equations, Science Press Beijing \& Springer-Verlag Berlin Heidelberg, Beijing, 2013. 
